\title{
Determination of Electron Swarm Parameters and Limiting Electrical Field during Martian Dust Storm Season Using Boltzmann Analysis
}

\author{
M.S. Dincer ${ }^{a}$, S.S. TezCan ${ }^{b, *}$, H. Duzkaya ${ }^{b}$ And S. Dincer $^{c}$ \\ ${ }^{a}$ Near East University, Department of Electrical-Electronics Engineering, \\ 99138, Nicosia, Cyprus \\ ${ }^{b}$ Gazi University, Department of Electrical-Electronics Engineering, 06570, Ankara, Turkey \\ ${ }^{c}$ Ankara University, Department of Chemistry, 06100, Ankara, Turkey \\ Received: 01.08.2019 \& Accepted: 29.04.2020
}

Doi: 10.12693/APhysPolA.138.368

*e-mail: $\quad$ stezcan@gazi.edu.tr

\begin{abstract}
In the ternary mixture of $95.7 \% \mathrm{CO}_{2}+2.69 \% \mathrm{~N}_{2}+1.59 \%$ Ar with various dust loads representing the Martian atmosphere, ionization, attachment, electron mean energies and electron drift velocities are numerically calculated by a two-term Boltzmann equation analysis at $L_{s}=270^{\circ}$. The attachment coefficient defined carries dissociative attachment collisions of the $\mathrm{CO}_{2}$ component in the ternary mixture together with electron attachment to uncharged dust grains in the dusty storm season. The limiting $E / N$ values for the critical condition where ionization rates are balanced by attachment collisions in the low pressure plasma ( $E$ is the electric field produced by the dust storm and $N$ is the total number density of gas molecules in the ternary mixture) are evaluated for various dust loads. The limiting $E / N$ increases as a function of dust grain size and the effect is more pronounced for dust loads with higher grain densities.
\end{abstract}

topics: martian atmosphere, electrical discharge, electron swarm properties, limiting electrical field, transport properties

\section{Introduction}

In situ measurements of electrical fields in the Martian atmosphere is not available up to date, although attempts, including the recent crash down of the Schiaparelli lander, which was supposed to search electrical effects in the Martian atmosphere with dust devils, have been made [1]. It is expected that dust storms in Mars may produce electric fields of high magnitude. Desert field tests, simulations and measurements carried out for terrestrial dust storms indicate that in the low pressure atmosphere of Mars, ambient breakdown strength of can be reached due to contact electrification of the dust grains producing the dust storm electrical field [2-6].

Critical field, $E$ in a dusty low pressure $\mathrm{CO}_{2}$ atmosphere has been defined before by setting the electron source term equal to the dust loss term [7]. If the electrical field produced by the dust storm is lower than the critical field, the dust uncharged in the storm is absorbing the electrons faster than electron impact ionizations and electron avalanche initiation is not possible. At the critical $E$ field, the rate of electron absorption due to dust is equal to ionization rate and any $E$ field value above the critical $E$ field will result in avalanche initiation due to the increased number of ionization collisions. However, in the previous work of the authors [7], the attachment process due to dissociative attachment of $\mathrm{CO}_{2}$ is ignored and avalanche growth is only controlled by uncharged dust electron absorption. The recent work of the authors defines dust devil avalanche model for the first time in literature which includes the dissociative attachment process, and recombination loss at high electric fields with the main interest focused on the evolution of the primary gaseous chemical species involved in the electrochemistry [8].

Experimental results carried out in 5 Torr of $\mathrm{CO}_{2}$ gas sample with insulating mesh sheets placed between electrodes to simulate dust absorption of free electrons, demonstrate that there is a reduction in the electron avalanche current together with increased spark discharge electric field compared to the case of dust free $\mathrm{CO}_{2}$ sample at the same pressure and gap setting [9]. The experimental findings of [9] also support the free electron loss due to electron absorption of uncharged dust in the dust storm.

Meteorological and seasonal phenomena in Martian atmosphere change concentration, dust density, and electrical fields in the atmosphere. Experimental and theoretical studies examining the effect of these changes on atmospheric electrical properties provide important findings in terms of design and optimization of devices used in Martian exploration missions [10, 11]. 
Seasonal changes in the mixtures

TABLE I of Martian atmosphere [10].

\begin{tabular}{c|l|c|c|c}
\hline \hline$L_{s}$ & \multicolumn{1}{|c|}{ Specificities } & $\mathrm{CO}_{2}[\%]$ & $\mathrm{N}_{2}[\%]$ & $\mathrm{Ar}[\%]$ \\
\hline $0 \circ$ & $\begin{array}{l}\text { northern hemisphere } \\
\text { spring equinox }\end{array}$ & 95.67 & 2.68 & 1.59 \\
\hline $71^{\circ}$ & $\begin{array}{l}\text { aphelion (largest } \\
\text { Sun-Martian distance) }\end{array}$ & 86.14 & 8.67 & 5.14 \\
\hline $90^{\circ}$ & $\begin{array}{l}\text { northern hemisphere } \\
\text { summer solstice }\end{array}$ & 81.83 & 11.35 & 6.74 \\
\hline $180^{\circ}$ & $\begin{array}{l}\text { northern hemisphere } \\
\text { autumn equinox } \\
\text { (dust storm season begins) }\end{array}$ & 89.47 & 6.59 & 3.91 \\
\hline $251^{\circ}$ & $\begin{array}{l}\text { perihelion (smallest } \\
\text { Sun-Martian distance) } \\
\text { (dust storm season) }\end{array}$ & 95.70 & 2.68 & 1.59 \\
\hline $270^{\circ}$ & $\begin{array}{l}\text { northern hemisphere } \\
\text { winter solstice } \\
\text { (dust storm season) }\end{array}$ & 95.70 & 2.69 & 1.59 \\
\hline
\end{tabular}

The aim of the present paper is to evaluate critical $E / N$ values in the mixture of $\mathrm{CO}_{2}+\mathrm{N}_{2}+\mathrm{Ar}$ representing the Martian atmosphere, where $E$ is the electric field produced by the dust storm and $N$ is the total number density of gas molecules in the ternary mixture. The critical $E / N$ value is the limiting field at which ionization and attachment rates equal to each other. The gas concentrations in the atmosphere vary depending on the proximity of Mars to the sun as can be seen from Table I [12].

The gas concentration in the atmosphere of Mars varies according to the distance and angle between the sun and Mars, the dust storm season and the location of the sample. The ratios used by GarciaCosio et al. [13] others for DC discharge experiments on Mars ionosphere are 95.3\%, 2.7\%, and $1.6 \%$ for $\mathrm{CO}_{2}, \mathrm{~N}_{2}$ and $\mathrm{Ar}$, respectively. Trainer et al. [14] accepted the rates of $95.1 \%( \pm 0.3), 2.59 \%$ $( \pm 0.06), 1.94 \%( \pm 0.04)$ for $\mathrm{CO}_{2}, \mathrm{~N}_{2}$ and Ar, respectively, at the Gale Crater as characteristic concentration data. This study concentrates on the electrical swarm parameters of the Martian atmosphere during the dust storm season, the ratios used in the study of Noguchi et al. [12], in which the concentration change in the Martian atmosphere in this period is described in detail and continuously. In this work, the ratios of $\mathrm{CO}_{2}, \mathrm{~N}_{2}$, and $\mathrm{Ar}$ in the ternary mixture are $95.7,2.69$, and 1.59 respectively corresponding to the dust storm season at $L_{s}=270^{\circ}$.

The limiting $E / N$ with the unit of $\mathrm{Td}(1 \mathrm{Td}=$ $10^{-21} \mathrm{~V} \mathrm{~m}^{2}$ ), namely townsend, is the critical field value at which the ionization rate and attachment rate in plasma are balanced. Therefore, the corresponding reduced effective ionization coefficient $(\alpha-$ $\eta) / N$ should be zero. The effective ionization coefficient is calculated from the Townsend ionization coefficient $\alpha$ and the electron attachment coefficient $\eta$. $\alpha$ and $\eta$ are evaluated from the ionization and attachment rate coefficients which are calculated from electron energy distributions based on the respective collision cross-section sets of ionization and attachment and number densities of all components in the gas mixture. The effective ionization coefficient is simply defined as $\alpha_{\text {eff }}=(\alpha-\eta)$, and $\eta$ is greater than $\alpha$ due to the dominant attachment processes for $E / N$ lower than the $(E / N)_{\text {limit }}$. Therefore, effective ionization coefficient will assume negative values and avalanche initiation and growth will not be possible at $E / N$ lower than the $(E / N)_{\text {limit }}$. Theoretical calculations and experimental measurements of effective ionization coefficients in various gases at $E / N$ lower than the limiting field and evaluation of the limiting fields have been reported in literature before by many researchers and by the present authors [15-18]. The $E / N$ values higher than the limiting $E / N$ will result in the initiation and growth of an electron avalanche.

In the present paper attachment collisions are governed by the dissociative attachment collision cross-section of the $\mathrm{CO}_{2}$ component in the ternary mixture together with the presented electron absorption collision cross-section of the dust load for various grain sizes and densities.

\section{Calculation method}

The swarm parameters of the $\mathrm{CO}_{2}, \mathrm{~N}_{2}$ and argon gas mixtures investigated in this study are numerically calculated using the numerical solutions of the Boltzmann equation with the two-term approach. Numerical solutions depend on the two-term extension of the electron energy distribution function, which is solved by the finite difference method as given as [19, 20]:

$$
\begin{aligned}
& \frac{E^{2}}{N^{2}} \frac{\mathrm{d}}{\mathrm{d} \varepsilon}\left(\frac{\varepsilon}{3 Q_{m}^{e}} \frac{\mathrm{d} f}{\mathrm{~d} \varepsilon}\right)+\frac{e E(\alpha-\eta)}{N^{2}} \frac{\mathrm{d}}{\mathrm{d} \varepsilon}\left(\frac{\varepsilon}{3 Q_{m}^{e}} f\right)+\frac{(\alpha-\eta)^{2}}{N^{2}} \frac{\varepsilon}{3 Q_{m}^{e}} f+\frac{2 m}{M} \frac{\mathrm{d}}{\mathrm{d} \varepsilon}\left(\varepsilon^{2} Q_{m} f\right) \\
& \quad+\left(\varepsilon+\varepsilon_{\nu}\right) Q_{\nu}\left(\varepsilon+\varepsilon_{\nu}\right) f\left(\varepsilon+\varepsilon_{\nu}\right)+\left(\varepsilon+\varepsilon_{e x}\right) Q_{e x}\left(\varepsilon+\varepsilon_{e x}\right) f\left(\varepsilon+\varepsilon_{e x}\right)-\varepsilon Q_{\nu}(\varepsilon) f(\varepsilon) \\
& -\varepsilon Q_{e x}(\varepsilon) f(\varepsilon)-\varepsilon Q_{i}(\varepsilon) f(\varepsilon)-\varepsilon Q_{a}(\varepsilon) f(\varepsilon)+\frac{1}{\Delta}\left(\frac{\varepsilon}{\Delta}+\varepsilon_{i}\right) Q_{i}\left(\frac{\varepsilon}{\Delta}+\varepsilon_{i}\right) f\left(\frac{\varepsilon}{\Delta}+\varepsilon_{i}\right) \\
& \quad+\frac{1}{1-\Delta}\left(\frac{\varepsilon}{1-\Delta}+\varepsilon_{i}\right) Q_{i}\left(\frac{\varepsilon}{1-\Delta}+\varepsilon_{i}\right) f\left(\frac{\varepsilon}{1-\Delta}+\varepsilon_{i}\right)=0
\end{aligned}
$$


where $e$ and $m$ are the electron charge and mass. $M$ is the molecular mass, $f$ is the electron energy distribution function, $\alpha, \eta$ and $\alpha-\eta$ are the ionization, attachment, and effective ionization coefficients, respectively. $E$ is the applied electrical field intensity, and $N$ is the total number density of the molecules in the gas mixture. $Q_{m}, Q_{v}, Q_{e x}, Q_{a}$, and $Q_{i}$ are the electron collision cross-section of momentum transfer, vibration, excitation, attachment, and ionization, respectively. $k_{\mathrm{CO}_{2}}, k_{\mathrm{N}_{2}}$ and $k_{\mathrm{Ar}}$ express the $\mathrm{CO}_{2}$ ratio, $\mathrm{N}_{2}$ ratio and argon ratio in the mixture, respectively. Therefore, electron collision cross-sections of the ternary mixture are defined as:

$$
\begin{aligned}
& Q_{m \text { mix }}=k_{\mathrm{CO}_{2}} Q_{m \mathrm{CO}_{2}}+k_{\mathrm{N}_{2}} Q_{m \mathrm{~N}_{2}} \\
& +k_{\mathrm{Ar}} Q_{m \mathrm{Ar}} \\
& Q_{v \text { mix }}=k_{\mathrm{CO}_{2}} Q_{v \mathrm{CO}_{2}}+k_{\mathrm{N}_{2}} Q_{v \mathrm{~N}_{2}}, \\
& Q_{e x \text { mix }}=k_{\mathrm{CO}_{2}} Q_{e x \mathrm{CO}_{2}}+k_{\mathrm{N}_{2}} Q_{e x \mathrm{~N}_{2}} \\
& +k_{\mathrm{Ar}} Q_{e x \mathrm{Ar}} \\
& Q_{a \text { mix }}=k_{\mathrm{CO}_{2}} Q_{a \mathrm{CO}_{2}} \\
& Q_{i \text { mix }}=k_{\mathrm{CO}_{2}} Q_{i \mathrm{CO}_{2}}+k_{N_{2}} Q_{i \mathrm{~N}_{2}} \\
& +k_{A r} Q_{i \mathrm{Ar}} \\
& Q_{m}^{e}=Q_{m \text { mix }}+Q_{\nu \text { mix }}+Q_{e x \text { mix }} \\
& +Q_{a \text { mix }}+Q_{i \text { mix }}
\end{aligned}
$$

The sum of these sections $\left(Q_{m}^{e}\right)$ is the momentum transfer effective collision section. Furthermore, in (1), $\varepsilon_{\nu}, \varepsilon_{e x}$, and $\varepsilon_{i}$ are the corresponding threshold energies of the vibration, excitation, and ionization collision cross-sections, respectively. After an ionization collision process, the remaining energy is shared equally between the primary and secondary electrons by assuming $\Delta$ to be equal to 0.5 . In (1), the kinetic energy of an electron at $u$ is defined as:

$$
\varepsilon=\frac{1}{2} m u^{2} .
$$

The electron drift velocity $W$, the reduced ionization coefficient $\alpha / N$ and the reduced attachment coefficient $\eta / N$ are calculated, respectively, using (9)-(12). The effect of dust is included to the attachment coefficient by adding the term calculated by (10), where $n_{d}$ is the uncharged dust grain density and $r_{d}$ is the radius of a single dust grain in the collisional plasma [7].

$$
\begin{aligned}
W & =-\frac{e}{3} \sqrt{\frac{2}{m}} \frac{E}{N} \int_{\varepsilon_{i}}^{\infty} \frac{\varepsilon}{Q_{m}^{e}} \frac{\partial f(\varepsilon)}{\partial \varepsilon} \mathrm{d} \varepsilon \\
\frac{\alpha}{N} & =\sqrt{\frac{2 e}{m}} \frac{1}{W} \int_{\varepsilon_{i}}^{\infty} \varepsilon Q_{i}(\varepsilon) f(\varepsilon) \mathrm{d} \varepsilon \\
\eta_{d} & =\pi n_{d} r_{d}^{2}
\end{aligned}
$$

$$
\frac{\eta}{N}=\sqrt{\frac{2 e}{m}} \frac{1}{W} \int_{\varepsilon_{a}}^{\infty} \varepsilon Q_{a}(\varepsilon) f(\varepsilon) \mathrm{d} \varepsilon+\frac{\eta_{d}}{N} .
$$

The collision cross-sections for $\mathrm{CO}_{2}$ are given by [21]. $\mathrm{N}_{2}$ collision cross-section set given by [22] is used. Argon collision cross-sections employed are presented by [23] and [24].

\section{Results and discussions}

In the present paper density normalized ionization coefficient, attachment coefficients, and electron mean energies and drift velocities are given as a function of $E / N$. The reduced ionization coefficients in $\mathrm{CO}_{2}$ calculated by Boltzmann equation analysis are compared with the Boltzmann [25, 26] and experimental results in the literature [27, 28], as shown in Fig. 1. The evaluated $\alpha / N$ agree very well with those of the experimental and theoretical values.

Figure 2 shows the change of reduced attachment coefficient in $\mathrm{CO}_{2}$ as a function of $E / N$. The results are consistent with the reported experimental and theoretical results in the literature in the $E / N$ interval of the present investigation.

The reduced effective ionization coefficient in $\mathrm{CO}_{2}$, which is the difference of reduced ionization coefficient and reduced attachment coefficient, is presented in Fig. 3. Compared with the theoretical and measurement results in the literature, the evaluated values are compatible with the reported values. The inset in the figure covers the $E / N$ interval from $100 \mathrm{Td}$ to $20 \mathrm{Td}$. As can be seen in Fig. 3, the effective ionization is zero in the vicinity of $78 \mathrm{Td}$ and the evaluated critical field which is the limiting $E / N$ is in agreement with the experimental values of [29].

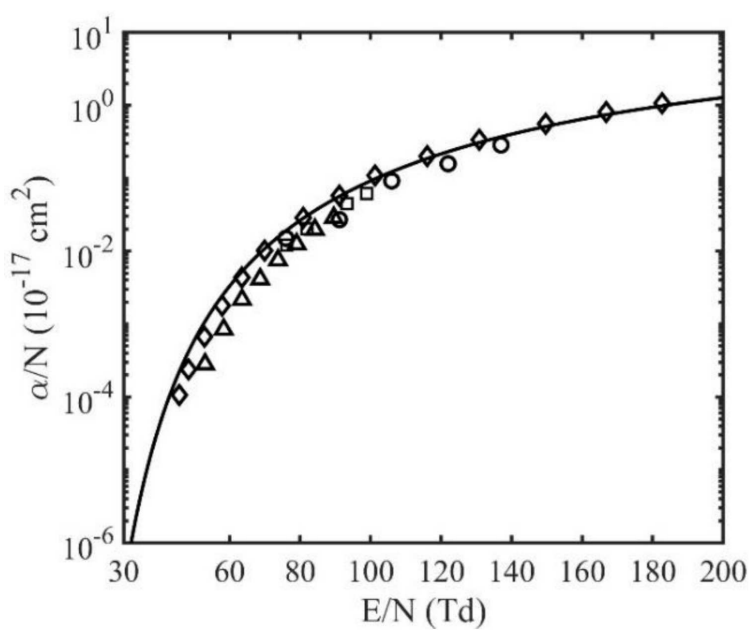

Fig. 1. The reduced ionization coefficients in $\mathrm{CO}_{2}$ : [25] Boltzmann results $(\triangle)$, [26] Boltzmann results $(\diamond),[27]$ experimental results $(\square),[28]$ experimental results, $(\bigcirc)$, present results (solid line). 


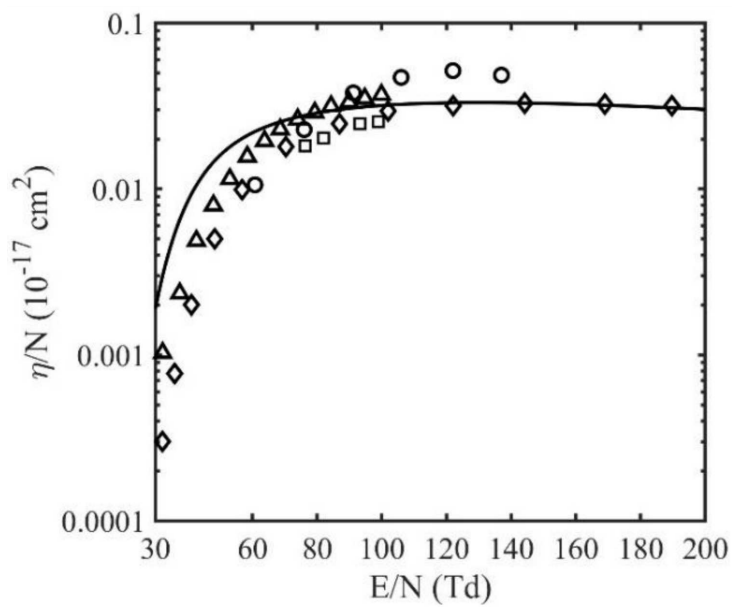

Fig. 2. As in Fig. 1, but for the reduced attachment coefficients in $\mathrm{CO}_{2}$.

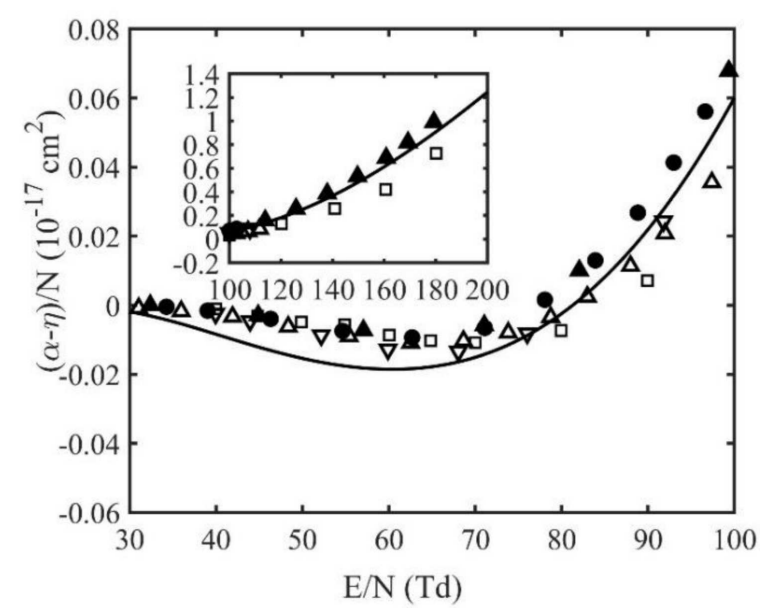

Fig. 3. The reduced effective ionization coefficients in $\mathrm{CO}_{2}$ [29] experimental results $(\triangle)$, [29] Magboltz results $(\nabla),[29]$ Bolsig + results $(\bullet),[30]$ experimental results $(\square),[26]$ Boltzmann results $(\mathbf{\Lambda})$, present results (solid line).

The electron drift velocity evaluated in $\mathrm{CO}_{2}$ is given in Fig. 4, as a function of $E / N$. As shown in Fig. 4, the value of the electron drift velocity increases as $E / N$ increases. There is reasonable agreement with those of the experimental measurements in the $E / N$ range of present investigation.

In Fig. 5, electron drift velocity in the Martian atmosphere at $L_{s}=270^{\circ}$ is shown together with the drift velocity of $\mathrm{CO}_{2}$. It can be observed in Fig. 5 that the drift velocity in the ternary mixture follows the same trend as a function of $E / N$ compared to the drift velocity in $\mathrm{CO}_{2}$, although the evaluated values in the ternary mixture are lower in the $E / N$ range.

The electron mean energies in pure $\mathrm{CO}_{2}$ and in the Martian atmosphere at $L_{s}=270^{\circ}$ are shown in Fig. 6. Both of the curves have the same trend as a function of $E / N$ although the mean energy in the ternary mixture assumes higher values in the $E / N$ interval of the present paper.

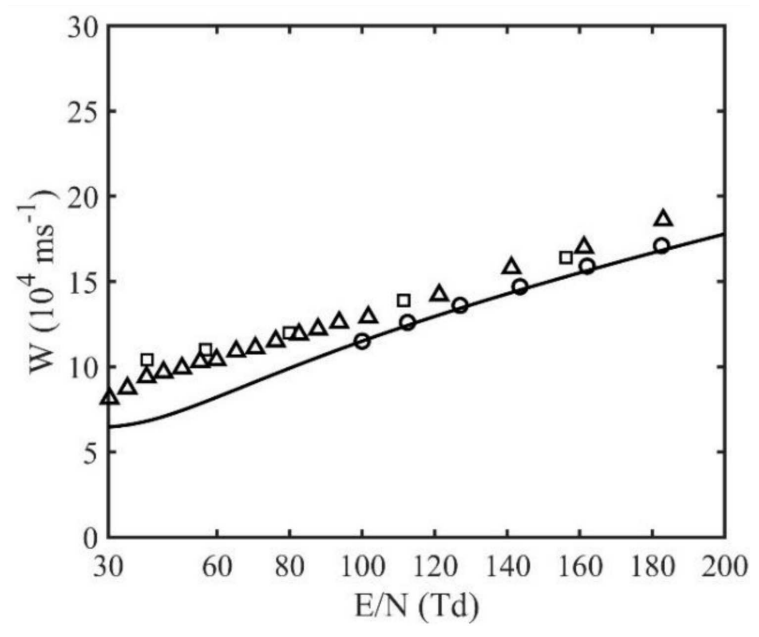

Fig. 4. Electron drift velocity in $\mathrm{CO}_{2},[31]$ experimental results $(\triangle),[32]$ Boltzmann results $(\bigcirc),[25]$ Boltzmann results $(\square)$, present results (solid line).

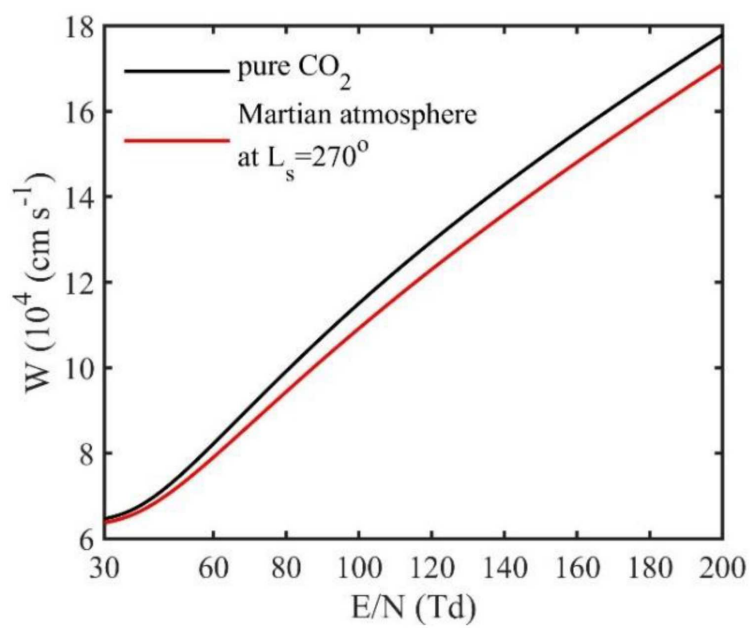

Fig. 5. The electron drift velocity in pure $\mathrm{CO}_{2}$ and Martian atmosphere at $L_{s}=270^{\circ}$.

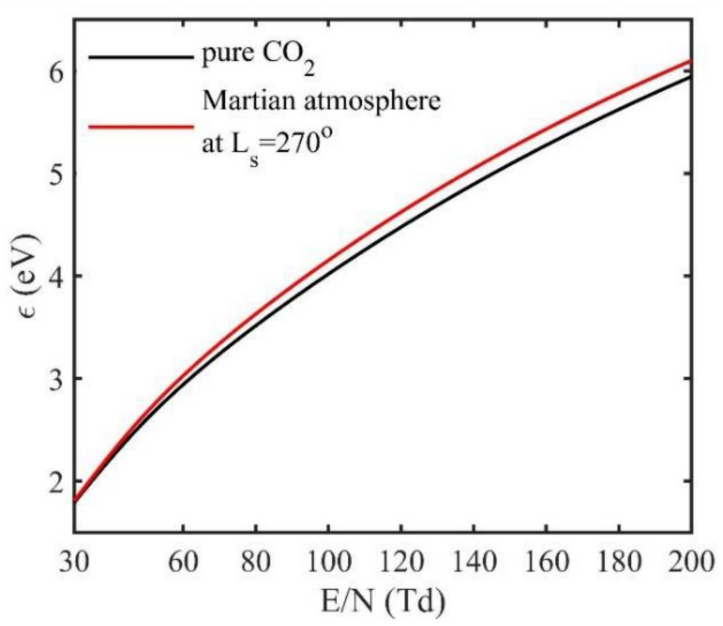

Fig. 6. The electron mean energy in pure $\mathrm{CO}_{2}$ and Martian atmosphere at $L_{s}=270^{\circ}$. 


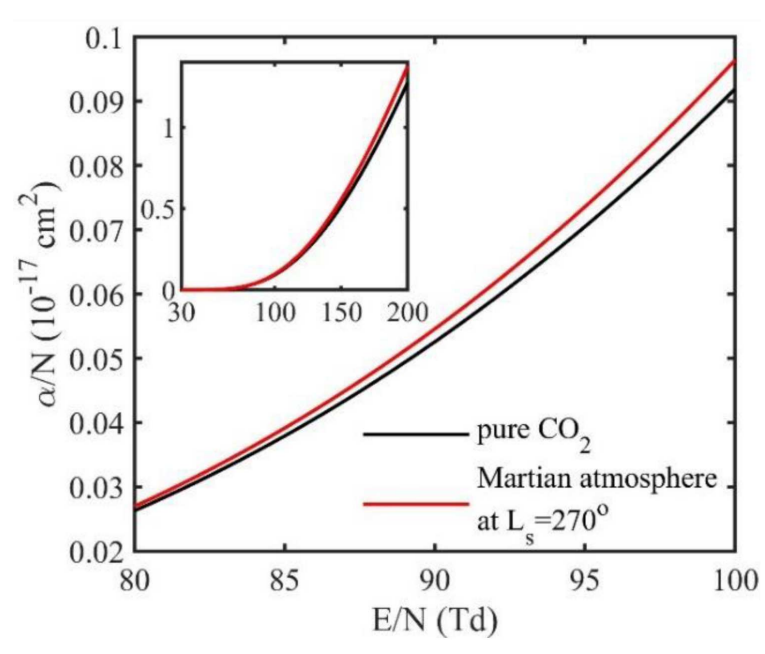

Fig. 7. The reduced ionization coefficients in pure $\mathrm{CO}_{2}$ and Martian atmosphere at $L_{s}=270^{\circ}$.

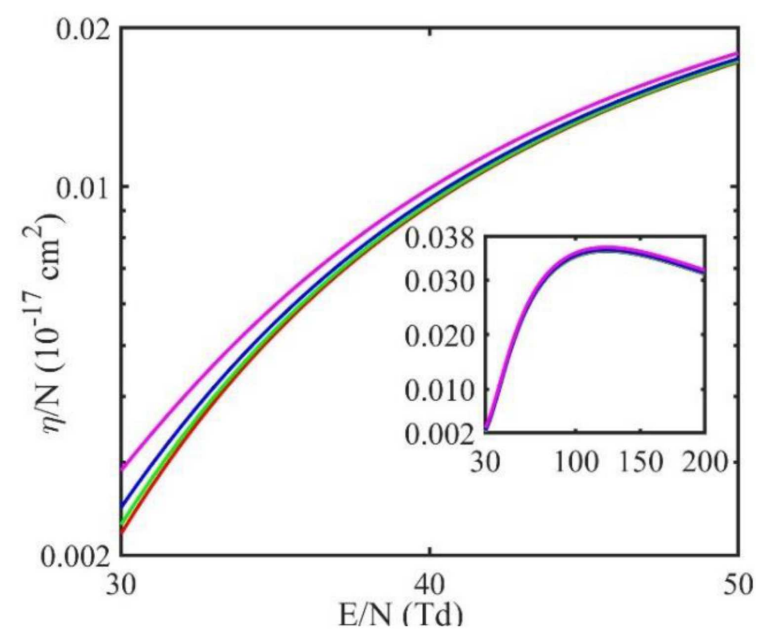

Fig. 8. The reduced attachment coefficients in the Martian atmosphere with the dust grain density of $5 \times 10^{6} \mathrm{~m}^{-3}$ at $L_{s}=270^{\circ}$ (red line $r_{D}=5 \times 10^{-6} \mathrm{~m}$, green line $r_{D}=15 \times 10^{-6} \mathrm{~m}$, blue line $r_{D}=25 \times$ $10^{-6} \mathrm{~m}$, magenta line $r_{D}=40 \times 10^{-6} \mathrm{~m}$ ).

Dust storms in the Martian atmosphere begin with the autumn equinox $\left(L_{s}=180^{\circ}\right.$; northern hemisphere autumn equinox) in the Arctic and dust storms continue until the first spring equinox $\left(L_{s}=360^{\circ}\right)$. The reduced ionization coefficients in the Martian atmosphere at $L_{s}=270^{\circ}$ is shown in Fig. 7 together with the ionization coefficient evaluated in $\mathrm{CO}_{2}$. It can be seen in Fig. 7 that the variation of the ionization coefficient as a function of $E / N$ for both curves has the same trend. The ionization coefficient evaluated in the Martian atmosphere is higher compared to that of $\mathrm{CO}_{2}$ for $E / N$ greater than $80 \mathrm{Td}$.

The dust phenomenon from dust storms in the Martian atmosphere directly changes the attachment coefficient, but the change in ionization coefficient is not present. Therefore, the ionization coefficient is given for dustless condition.

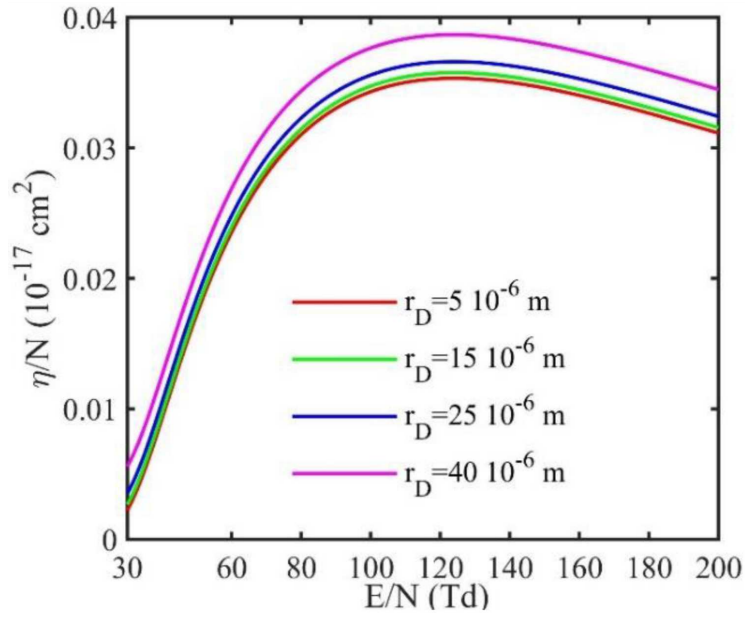

Fig. 9. The reduced attachment coefficients in the Martian atmosphere with the dust grain density of $24 \times 10^{6} \mathrm{~m}^{-3}$ at $L_{s}=270^{\circ}$.

Since the aim of the present paper is to analyse the effect of the dust load on the limiting $E / N$, a systematic analysis with various dust loads are considered for different dust grain sizes at a given grain density. The electron absorption of an uncharged dust grain is considered as an attachment mechanism with the presented collision crosssection defined by the grain radius and grain density. Hence the attachment coefficient $\eta / N$ evaluated carries dissociative attachment collisions of the $\mathrm{CO}_{2}$ component in the ternary mixture together with electron attachment to uncharged dust grains in the dusty storm season.

The reduced attachment coefficients in the Martian atmosphere with the dust grain density of $5 \times 10^{6} \mathrm{~m}^{-3}$ at $L_{s}=270^{\circ}$ is shown in Fig. 8 . The attachment coefficient increases with increasing radius of the dust grain in the $E / N$ range of 30-50 Td, however the contribution of the dust load is negligible above $50 \mathrm{Td}$ as can be seen from the inset in Fig. 8.

As the dust grain density increases, the attachment coefficient increases at a given $E / N$ and given grain radius as can be observed in Figs. 9-14 in the investigated $E / N$ range from $30 \mathrm{Td}$ to $200 \mathrm{Td}$. All the $\eta / N$ curves in the figures assume a peak value in the vicinity of $120 \mathrm{Td}$ and for $E / N$ above $120 \mathrm{Td}$ reduction in the attachment coefficient is observed. The variation in the attachment coefficient as a function of $E / N$ is due to the energy dependent dissociative attachment collision cross-section of the $\mathrm{CO}_{2}$ component in the ternary mixture.

From the attached curves in Figs. 8-14 it can be seen that at a given dust grain density the attachment coefficient increases as the dust grain radius is increased. The response to the dust load is more pronounced with increasing grain density since the collision cross-section of electron absorption presented to the incoming electrons increases with dust grain density. In the present paper, 


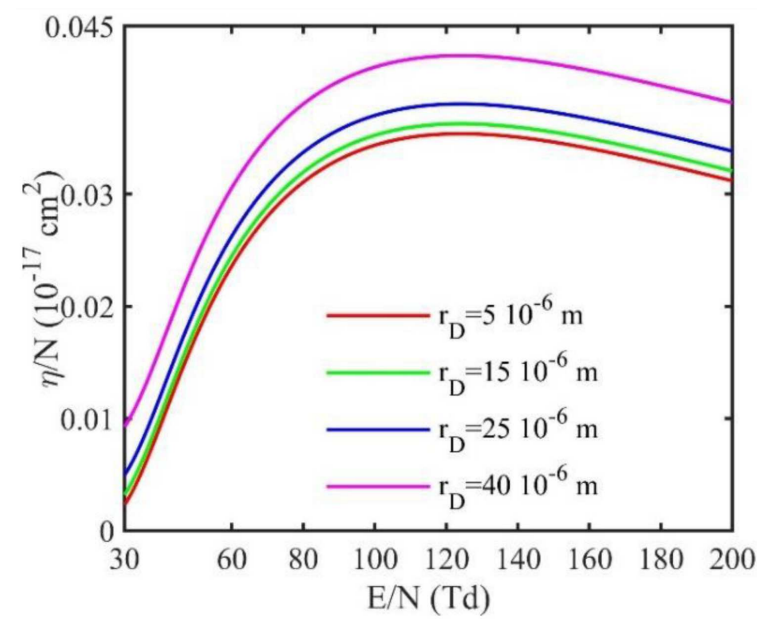

Fig. 10. The reduced attachment coefficients in the Martian atmosphere with the dust grain density of $50 \times 10^{6} \mathrm{~m}^{-3}$ at $L_{s}=270^{\circ}$.

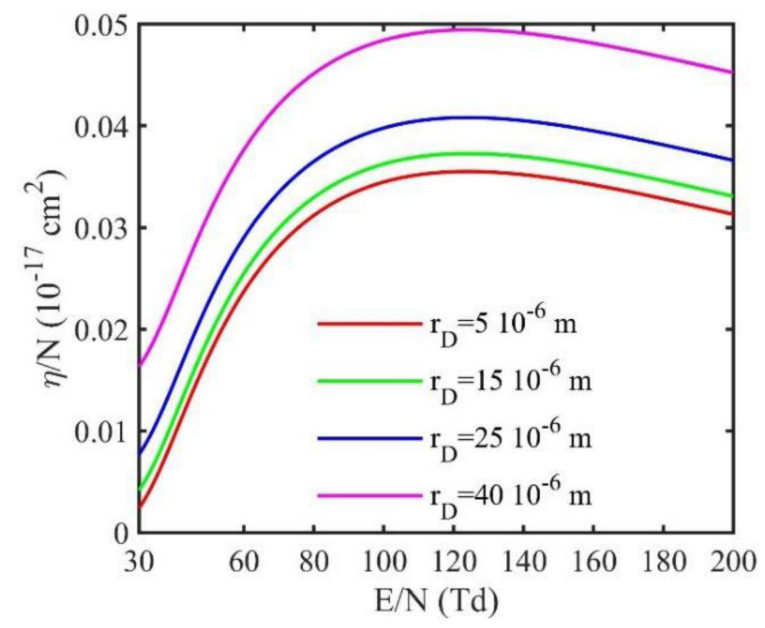

Fig. 11. The reduced attachment coefficients in the Martian atmosphere with the dust grain density of $100 \times 10^{6} \mathrm{~m}^{-3}$ at $L_{s}=270^{\circ}$.

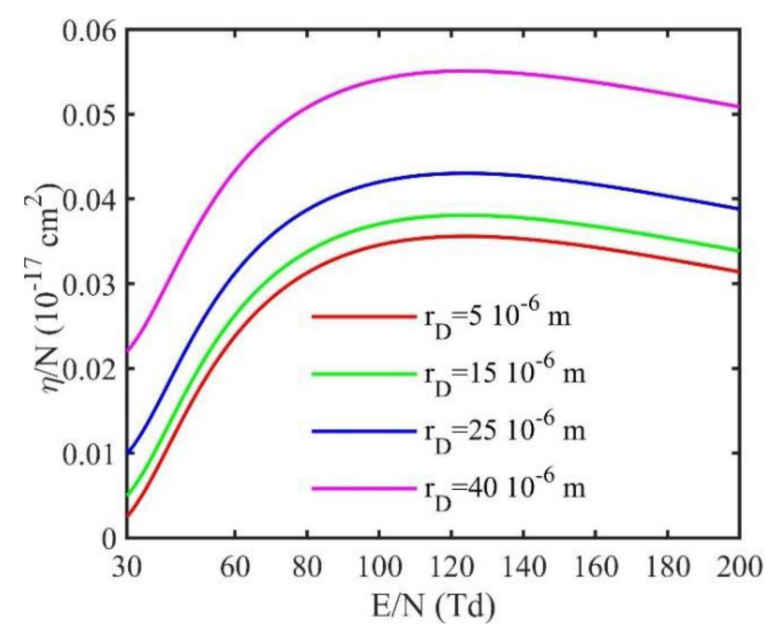

Fig. 12. The reduced attachment coefficients in the Martian atmosphere with the dust grain density of $140 \times 10^{6} \mathrm{~m}^{-3}$ at $L_{s}=270^{\circ}$.

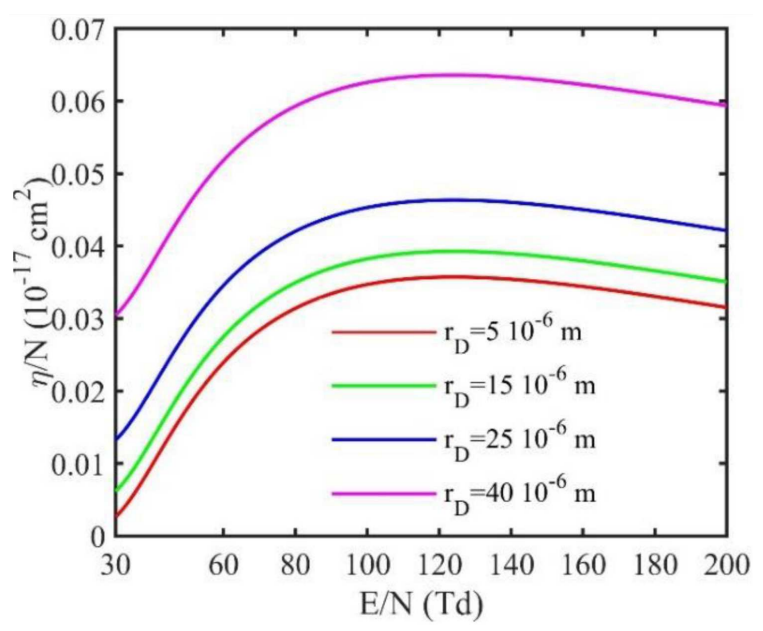

Fig. 13. The reduced attachment coefficients in the Martian atmosphere with the dust grain density of $200 \times 10^{6} \mathrm{~m}^{-3}$ at $L_{s}=270^{\circ}$.

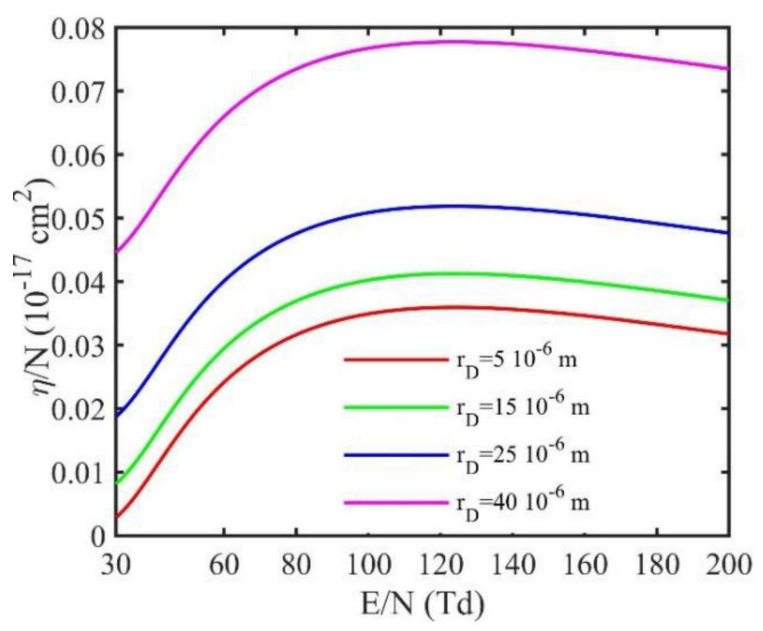

Fig. 14. The reduced attachment coefficients in the Martian atmosphere with the dust grain density of $300 \times 10^{6} \mathrm{~m}^{-3}$ at $L_{s}=270^{\circ}$.

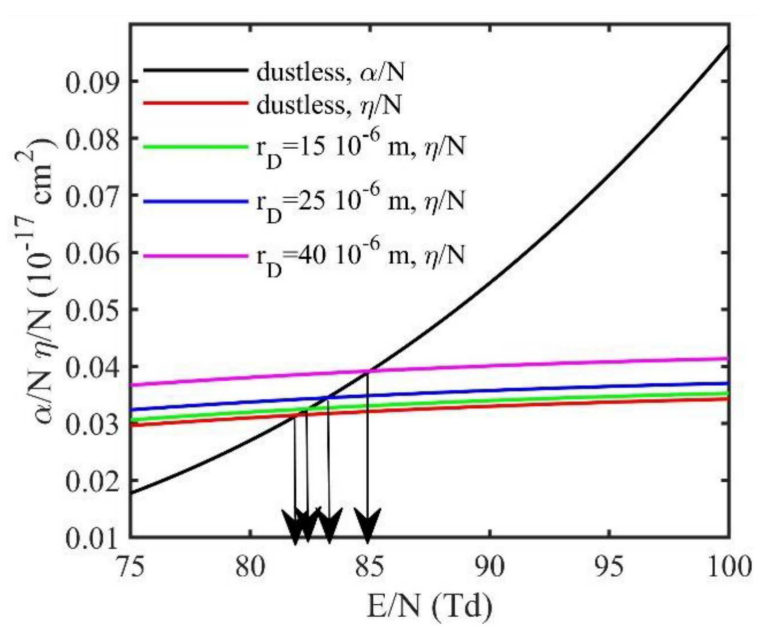

Fig. 15. The representation of finding the limiting electrical field in the Martian atmosphere with the dust grain density of $50 \times 10^{6} \mathrm{~m}^{-3}$ at $L_{s}=270^{\circ}$. 
TABLE II

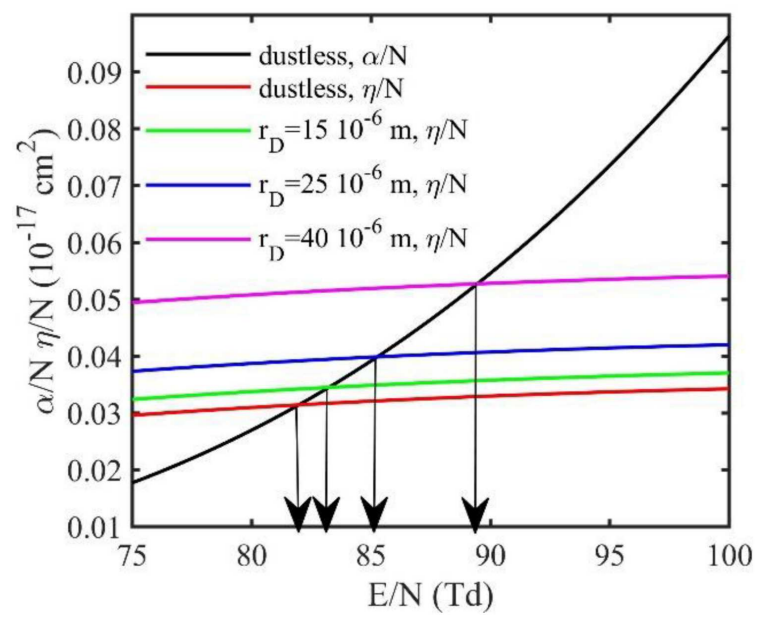

Fig. 16. The representation of finding the limiting electrical field in the Martian atmosphere with the dust grain density of $140 \times 10^{6} \mathrm{~m}^{-3}$ at $L_{s}=270^{\circ}$.

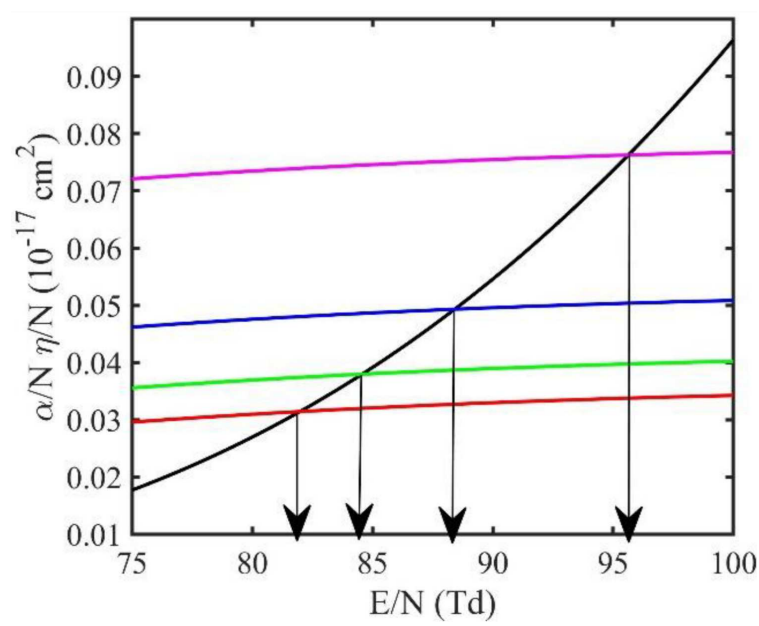

Fig. 17. The representation of finding the limiting electrical field in the Martian atmosphere with the dust grain density of $300 \times 10^{6} \mathrm{~m}^{-3}$ at $L_{s}=270^{\circ}$. The same colours are used for the same conditions as in Fig. 16.

dust grain densities are considered as $5 \times 10^{6} \mathrm{~m}^{-3}$, $24 \times 10^{6} \mathrm{~m}^{-3}, 50 \times 10^{6} \mathrm{~m}^{-3}, 100 \times 10^{6} \mathrm{~m}^{-3}, 140 \times$ $10^{6} \mathrm{~m}^{-3}, 200 \times 10^{6} \mathrm{~m}^{-3}$, and $300 \times 10^{6} \mathrm{~m}^{-3}$ with the grain radius varying from $0.75 \times 10^{-6} \mathrm{~m}$ to $40 \times 10^{-6} \mathrm{~m}$. In the parameter range of the present paper, it is observed that for grain size less than a radius of $5 \times 10^{-6} \mathrm{~m}$, the dust load effect is not significant in the calculations and attachment coefficient is mainly governed by dissociative attachment collisions.

The reduced ionization coefficient and the reduced attachment coefficient are plotted in a single graph as a function of $E / N$ at the given dust load condition and then the $E / N$ value at the point where their intersect is obtained. The intersection point yields the critical $E / N$ value known as the limiting $E / N$ at which the effective ionization coefficient is zero since $\alpha / N=\eta / N$. The representation
The limiting electrical field, the electron mean energy and the electron drift velocity in the Martian atmosphere with typical dust grain radius and density at $L_{s}=270^{\circ}$.

\begin{tabular}{c|c|c|c|c|c|c}
\hline \hline $\begin{array}{c}r_{D} \\
{[\mu \mathrm{m}]}\end{array}$ & $\begin{array}{c}E / N_{\text {limit }} \\
{[\mathrm{Td}]}\end{array}$ & $\varepsilon[\mathrm{eV}]$ & $\begin{array}{c}W \\
{\left[10^{4} \mathrm{~m} / \mathrm{s}\right]}\end{array}$ & $\begin{array}{c}E / N_{\text {limit }} \\
{[\mathrm{Td}]}\end{array}$ & $\varepsilon[\mathrm{eV}]$ & $\begin{array}{c}W \\
{\left[10^{4} \mathrm{~m} / \mathrm{s}\right]}\end{array}$ \\
\hline & \multicolumn{2}{|c|}{$n_{D}=5 \times 10^{6} \mathrm{~m}^{-3}$} & \multicolumn{2}{|c|}{$n_{D}=50 \times 10^{6} \mathrm{~m}^{-3}$} \\
\hline 0.75 & 81.98 & 3.6840 & 9.5882 & 81.98 & 3.6837 & 9.5882 \\
2 & 81.98 & 3.6840 & 9.5882 & 81.99 & 3.6842 & 9.5890 \\
5 & 81.99 & 3.6842 & 9.5890 & 82.03 & 3.6853 & 9.5919 \\
10 & 82.00 & 3.6845 & 9.5897 & 82.19 & 3.6898 & 9.6040 \\
15 & 82.03 & 3.6853 & 9.5920 & 82.44 & 3.6967 & 9.6227 \\
25 & 82.11 & 3.6876 & 9.5980 & 83.22 & 3.7181 & 9.6810 \\
40 & 82.31 & 3.6931 & 9.6129 & 84.98 & 3.7662 & 9.8121 \\
\hline & $n_{D}=140 \times 10^{6} \mathrm{~m}^{-3}$ & $n_{D}=300 \times 10^{6} \mathrm{~m}^{-3}$ \\
\hline 0.75 & 81.99 & 3.6842 & 9.5890 & 81.99 & 3.6842 & 9.5890 \\
2 & 82.01 & 3.6848 & 9.5905 & 82.03 & 3.6853 & 9.5920 \\
5 & 82.13 & 3.6881 & 9.5995 & 82.29 & 3.6925 & 9.6114 \\
10 & 82.55 & 3.6997 & 9.6309 & 83.17 & 3.7168 & 9.6773 \\
15 & 83.23 & 3.6939 & 9.6136 & 84.55 & 3.7553 & 9.7824 \\
25 & 85.24 & 3.7733 & 9.8314 & 88.36 & 3.8573 & 10.0613 \\
40 & 89.39 & 3.8848 & 10.1366 & 95.49 & 4.0447 & 10.5756
\end{tabular}

of finding the limiting electrical field in the Martian atmosphere with different grain densities at $L_{s}=270^{\circ}$ is shown in Figs. 15, 16, and 17, respectively. The arrows in the respective figures point out the limiting electrical field.

The limiting electrical fields evaluated in the Martian atmosphere with typical dust loads of various densities at $L_{s}=270^{\circ}$ are given in Table II together with the corresponding electron mean energies and drift velocities. It can be observed from this table that as dust grain density increases, the limiting electrical field increases at a given grain size for particular radius greater than $5 \mu \mathrm{m}$. The effect of the dust load is more pronounced for larger dust grains with higher densities. In Table II, dust grain density of $140 \times 10^{6} \mathrm{~m}^{-3}$ corresponds to the expected dust storm density calculated using the MER optical data reported by [33].

\section{Conclusions}

The ionization and attachment coefficients, electron mean energies and electron drift velocities are evaluated as a function of reduced $E / N$ in the $95.7 \% \mathrm{CO}_{2}+2.69 \% \mathrm{~N}_{2}+1.59 \%$ Ar ternary mixture representing the Martian atmosphere at dusty storm season $\left(L_{s}=270^{\circ}\right)$. The attachment coefficient for various dust loads are evaluated considering dissociative attachment mechanism in the $\mathrm{CO}_{2}$ component of the ternary mixture together with electron attachment to uncharged dust grains. The limiting $E / N$ values are evaluated for various dust loads in the ternary mixture.

The limiting $E / N$ is an important parameter for the initiation of electron avalanche growth, which may turn into a Townsend spark discharge or even 


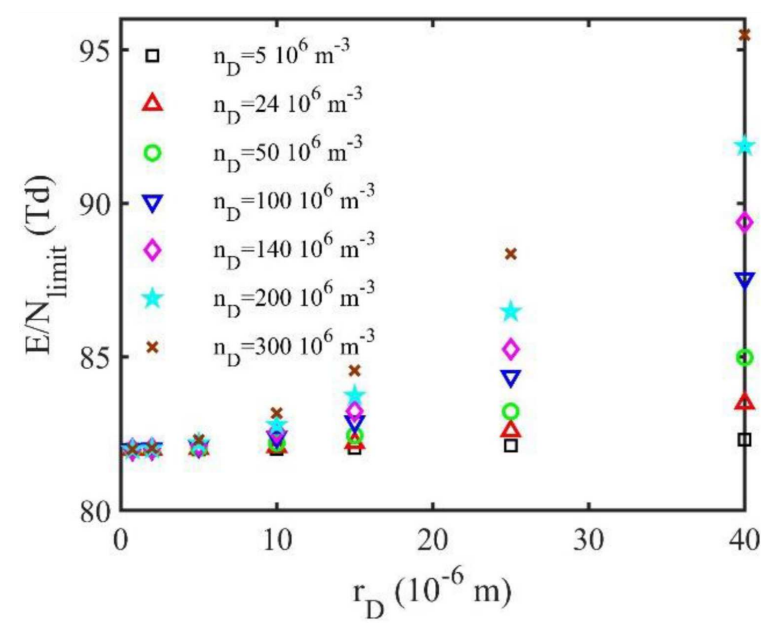

Fig. 18. The limiting electrical field in the Martian atmosphere with different dust grain radius and density at $L_{s}=270^{\circ}$.

under favourable conditions of space charge field built up supporting the dust storm electric field, may evolve into a streamer discharge.

It can be observed in Fig. 18 that the limiting $E / N$ increases as the dust grain size increases and the effect is more pronounced for dust loads with higher grain densities.

Whichever the type of discharge is, corona, partial, Townsend or streamer, the limiting $E / N$ evaluated with typical dust loads yields the condition of avalanche initiation which can result in breakdown and damage to hardware, and instrumentation of landers and rovers. It is generally accepted that electrical discharge is one of the risks for the landers and rovers [34].

The swarm data presented can help for possible implications for instrumentation and insulation design of future missions. Furthermore, electrical discharge is considered as a possible source for methane production on Mars [35].

\section{References}

[1] R.G. Harrison, E. Barth, F. Esposito et al., Space Sci. Rev. 203, 299 (2016).

[2] O. Melnik, M. Parrot, J. Geophys. Res. 103, 107 (1998).

[3] W.M. Farrell, G.T. Delory, S.A. Cummer, J.R. Marshall, Geophys. Res. Lett. 30, 2050 (2003).

[4] W.M. Farrell, N. Renno, G.T. Delory, S.A. Cummer, J.R. Marshall, J. Geophys. Res. 111, E01006 (2006).

[5] Y. Zhai, S.A. Cummer, W.M. Farrell, J. Geophys. Res. 111, E06016 (2006).

[6] T.L. Jackson, W.M. Farrell, IEEE Trans. Geosci. Rem. Sens. 44, 2942 (2006).
[7] T.L. Jackson, W.M. Farrell, G.T. Delory, J. Nithianandam, J. Geophys. Res. Lett. 35, L16201 (2008).

[8] T.L. Jackson, W.M. Farrell, G.T. Delory, J. Nithianandam, J. Geophys. Res. 115, E05006 (2010).

[9] W.M. Farrell, J.L. McLain, M.R. Collier, J.W. Keller, Icarus 297, 90 (2017).

[10] G. Franzese, F. Esposito, R. Lorenz et al., Earth Planet. Sci. Lett. 493, 71 (2018).

[11] E.L. Barth, W.M. Farrell, S.C.R. Rafkin, Icarus 268, 253 (2016.

[12] K. Noguchi, S. Ikeda, T. Kuroda, S. Tellmann, M. Patzold, J. Geophys. Res. Planets 119, 2510 (2014).

[13] G. Garcia-Cosio, H. Martinez, M. CalixtoRodriguez, A. Gomez, J. Quant. Spectrosc. Radiat. Transfer 112, 2787 (2011).

[14] M.G. Trainer, M.H. Wong, T.H. McConnochie et al., J. Geophys. Res. Planets 124, 3000 (2019).

[15] J. de Urquijo, A. Mitrani, G. Ruiz-Vargas, E. Basurto, J. Phys. D Appl. Phys. 44, 342001 (2011).

[16] H.E. Nechmi, A. Beroual, A. Girodet, P. Vinson, IEEE Trans. Dielectr. Electr. Insul. 24, 886 (2017).

[17] S.S. Tezcan, H. Duzkaya, S. Dincer, M.S. Dincer, Acta Phys. Pol. A 135, 495 (2019).

[18] M.S. Dincer, G.R.G. Raju, IEEE Trans. Electr. Insul. 19, 40 (1984).

[19] S.S. Tezcan, M.S. Dincer, S. Bektas, H.R. Hiziroglu, IEEE Trans. Dielectr. Electr. Insul. 20, 98 (2013).

[20] S.S. Tezcan, H. Duzkaya, M.S. Dincer, H.R. Hiziroglu, IEEE Trans. Dielectr. Electr. Insul. 23, 1996 (2016).

[21] LXCat, Biagi-v7.1 database, 2010.

[22] Y. Itikawa, J. Phys. Chem. Ref. Data 35, 31 (2006).

[23] M. Hayashi, National Institute for Fusion Science NIFS Data 072, 2003.

[24] A. Yanguas-Gil, J. Cotrino, L.L. Alves, J. Phys. D Appl. Phys. 38, 1588 (2005).

[25] Y. Deng, C. Lu, D. Xiao, IEEE Trans. Plasma Sci. 40, 2671 (2012).

[26] H. Zhao, Y. Deng, H. Lin, AIP Adv. 7, 095102 (2017).

[27] D.K. Davies, J. Appl. Phys. 49, 127 (1978).

[28] S.R. Alger, J.A. Rees, J. Phys. D Appl. Phys. 9, 2359 (1976).

[29] D.A. Dahl, T.H. Teich, C.M. Franck, J. Phys. D Appl. Phys. 45, 485201 (2012). 
[30] M. Yousfi, J. Urquijo, A. Juarez, E. Basurto, J.L. Hernandez-Avila, IEEE Trans. Plasma Sci. 37, 764 (2009).

[31] J.L. Hernandez-Avila, E. Basurto, J. Urquijo, J. Phys. D Appl. Phys. 35, 2264 (2002).

[32] D. Yun-Kun, X. Deng-Ming, Chin. Phys. B 22, 035101 (2013).

[33] C.I. Calle, P.J. Mackey, M.R. Johansen, J. III Phillips, R.E. Cox, NASA Technical Report Server, ID: 20160013317, 2016.
[34] H.L.K. Manning, I.L. ten Kate, S.J. Battel, P.R. Mahaffy, Adv. Space Res. 46, 1334 (2010).

[35] A. Robledo-Martinez, H. Sobral, A. RuizMeza, J. Geophys. Res. Lett. 39, L17202 (2012). 